\title{
EFFECT OF IMPURITIES ON DYNAMIC RECRYSTALLIZATION IN COPPER DEFORMED IN BRIDGMAN ANVILS
}

\author{
D. K. Orlova, T. I. Chashchukhina, L. M. Voronova, M. V. Degtyarev, Yu. G. Krasnoperova \\ M.N. Miheev Institute of Metal Physics of Ural Branch of Russian Academy of Sciences, 18 S. Kovalevskoy st., \\ Ekaterinburg, Russian Federation \\ *Corresponding author e-mail: highpress@imp.uran.ru; address for correspondence: 18, ul. S. Kovalevskoy, 620990, \\ Ekaterinburg, Russian Federation. Tel.: +7 3433783818
}

The structure of copper with a various content of impurities $(0.01,0.03$, and $0.1 \mathrm{wt} \%)$ is studied after high pressure torsion at room temperature. It is shown that the content of impurities does not affect the true strain $(e=2)$ required for the onset of dynamic recrystallization $(D R)$. It has been revealed that the temperature-strain-rate conditions $(\operatorname{lnZ})$, which were the same for copper of various purity, are responsible for the degree of the dynamic recrystallization completeness and the respective type of the formed structure. The correspondence between the range of $\ln Z$ and the structure type formed during deformation has been established. Impurity dragging prevents grain growth upon postdynamic recrystallization and ensures the formation of a more dimensionally uniform structure at the stage of partial DR. At the stage of developed DR, the content of impurities in copper does not affect the grain size.

Keywords: severe plastic deformation, copper, temperature-compensated strain rate, dynamic recrystallization, postdynamic recrystallization, structure.

DOI: $10.17804 / 2410-9908.2015 .5 .090-098$

\section{References}

1. Valiev R.Z., Aleksandrov I.V. Nanostrukturnye materialy, poluchennye intensivnoi plasticheskoi deformatsiei [Nanostructured Materials Produced by Severe Plastic Deformation]. Moscow, Logos Publ., 2000, 272p. (In Russian).

2. Andrievskiy R.A., Glezer A.M. Size effects in nanocrystalline materials: II. Mechanical and physical properties. Physics of Metals and Metallography, 2000, vol. 89, iss. 1, pp. 83-102.

3. Teplov V.A., Pilyugin V.P., Taluts G.G. Formation of dissipative structures and phase transitions in iron alloys upon shear under pressure. Izvestiya RAN. Metally, 1992, no. 2, pp. 109-115. (In Russian).

4. Noskova N.I., Mulyukov R.R. Submikrokristallicheskie $i$ nanokristallicheskie metally $i$ splavy [Submicrocrystalline and Nanocrystalline Metals and Alloys]. Ekaterinburg, UB RAS Publ., 2003, 279 p. (In Russian).

5. Popova E.N., Deryagina I.L., Valova-Zaharevskaya E.G., Stolbovsky A.V., Khlebova N.E., Pantsyrny V.I. Specific Features of Interfaces in $\mathrm{Cu}-\mathrm{Nb}$ Nanocomposites. Defect and Diffusion Forum, 2014, vol. 354, pp. 183-188. DOI: 10.4028/www.scientific.net/DDF.354.183.

6. Degtyarev M.V., Chashchukhina T.I., Voronova L.M., Patselov A.M., Pilyugin V.P. Influence of the relaxation processes on the structure formation in pure metals and alloys under high-pressure deformation. Acta Materialia, 2007, vol. 55, pp. 6039-6050. DOI: $10.1016 /$ j.actamat.2007.04.017.

7. Kaigorodova L.I., Rasposienko D.Yu., Pushin V.G., Pilyugin V.P., Smirnov S.V. Structure and mechanical properties of aging $\mathrm{Al}-\mathrm{Li}-\mathrm{Cu}-\mathrm{Zr}-\mathrm{Sc}-\mathrm{Ag}$ alloy after severe plastic deformation by high-pressure torsion. Physics of Metals and Metallography, 2015, vol.116, iss. 4, pp. 346-355. DOI: $10.1134 / \mathrm{S} 0031918 \mathrm{X} 15040080$.

8. Shabashov V.A., Korshunov L.G., Sagaradze V.V., Kataeva N.V., Zamatovsky A.E., Litvinov A.V., Lyashkov K.A. Mossbauer analysis of deformation dissolution of the products of 
cellular decomposition in high-nitrogen chromium manganese austenite steel. Philosophical Magazine, 2014, vol. 94, pp. 668-682. DOI: 10.1080/14786435.2013.859758.

9. Zel'dovich V.I., Frolova N.Yu., Khomskaya I.V., Kheifets A.E., Shorokhov E.V., Nasonov P.A. Structure and microhardness of chromium-zirconium bronze subjected to severe plastic deformation by dynamic channel-angular pressing and rolling. Physics of Metals and Metallography, 2014, vol. 115, iss. 5, pp. 465-470. DOI: 10.1134/S0031918X14050159.

10. Brodova I.G., Shirinkina I.G., Petrova A.N., Pilyugin V.P., Tolmachev T.P. Structure of an AMts aluminum alloy after high-pressure torsion in liquid nitrogen. Physics of Metals and Metallography, 2013, vol. 114, iss. 8, pp. 667-671. DOI: 10.1134/S0031918X13080024.

11. Smirnova N.A., Levit V.I., Pilyugin V.P., Kuznetsov R.I., Degtyarev M.V. Features of lowtemperature recrystallization of nickel and copper. Physics of Metals and Metallography, 1986, vol. 62 , iss. 3 , pp. $140-144$.

12. Smirnov A.S., Konovalov A.V., Muizemnek O.Yu. Modelling and Simulation of Strain Resistance of Alloys Taking into Account Barrier Effects. Diagnostics, Resource and Mechanics of materials and structures, 2015, iss. 1, pp. 61-72. Available at: URL: $\quad$ http://dream-journal.org/issues/2015-1/2015-1_18.html (accessed: 10.09.2015). DOI: $10.17804 / 2410-9908.2015 .1 .061-072$.

13. Chashchukhina T.I., Degtyarev M.V., Romanova M.Yu., Voronova L.M. Dynamic recrystallization in copper deformed by shear under pressure. Physics of Metals and Metallography, 2004, vol. 98, pp. 639-647.

14. Bernshtein M.L., Dobatkin S.V., Kaputkina L.M., Prokoshkin S.D. Diagrammy goryachey deformatsii, struktura i svoistva staley [Hot Strain Diagrams, the Structure and Properties of Steels]. Moscow, Metallurgiya Publ, 1989, 544 p. (In Russian).

15. Levit V.I., Smirnov M.A. Vysokotemperaturnaya termomekhanicheskaya obrabotka austenitnykh staley i splavov [High-Temperature Thermomechanical Treatment of Austenitic Steels and Alloys]. Chelyabinsk CSTU Publ., 1995, 276 p. (In Russian).

16. Gorelik S.S. Rekristallisatsiya metallov i splavov [Recrystallization of Metals and Alloys]. Moscow, Metallurgiya Publ., 1978, 568 p. (In Russian).

17. Degtyarev M.V., Chashchukhina T.I., Voronova L.M., Kopylov V.I. Establishment of Conformity between the true strain, hardness and size of the structural elements of iron and structural steel under severe plastic deformation in different ways. Fizicheskaya mezomekhanika, 2013, vol. 16, no. 6, pp. 71-80. (In Russian).

18. McQeen H. J. The production and utility of recovered dislocation substructures. Metallurgical Transactions A, 1977, vol. 8, iss. 6, pp. 807-824. DOI: 10.1007/BF02661562.

19. Amirkhanov N.M., Islamgaliev R.K., Valiev R.Z. Thermal relaxation and grain growth upon isothermal annealing of ultrafine_grained copper produced by severe plastic deformation. Physics of Metals and Metallography, 1998, vol. 86, iss. 3, pp. 296-301.

20. Saltykov S.A. Stereometricheskaya metallografiya [Stereometric Metallography]. Moscow, Metallurgiya Publ., 1970, 375p. (In Russian).

21. Novikov V.Yu. Vtorichnaya rekristallizatsiya [Secondary Recrystallization]. Moscow, Metallurgiya Publ., 1990, 128p. (In Russian). 
Подана в журнал: 15.09 .2015

УДК 539.374.2:620.186.5:669-174:669.3

DOI: $10.17804 / 2410-9908.2015 .5 .090-098$

\title{
ВЛИЯНИЕ ПРИМЕСЕЙ НА ДИНАМИЧЕСКУЮ РЕКРИСТАЛЛИЗАЦИЮ В МЕДИ, ДЕФОРМИРОВАННОЙ В НАКОВАЛЬНЯХ БРИДЖМЕНА
}

\author{
Д. К. Орлова, Т. И. Чащухина, Л. М. Воронова, М. В. Дегтярев, Ю. Г. Красноперова \\ Федеральное государственное бюджетное учреждение науки Институт физики металлов имени \\ М.Н. Михеева Уральского отделения Российской академии наук, ул. С. Ковалевской, 18, Екатеринбург, \\ Российская Федеращия
}

\begin{abstract}
*Ответственный автор. Электронная почта: highpress@imp.uran.ru; адрес для переписки: ул. С.Ковалевской, 18, 620990, Екатеринбург, Российская Федерация. Телефон: +7 (343) 378-38-18
\end{abstract}

Проведено исследование структуры меди с различным $(0,01 ; 0,03$ и 0,1 мас. \%) содержанием примесей, деформированной при комнатной температуре методом сдвига под давлением. Показано, что количество примесей не влияет на величину деформации (е = 2), необходимой для начала динамической рекристаллизации (ДР). Установлено, что полнота протекания динамической рекристаллизации и соответственно тип формирующейся структуры определяются температурно-скоростными условиями деформации $(\ln Z)$, одинаковыми для меди разной степени чистоты. Выявлены интервалы $\ln Z$, отвечающего за тип структуры, формирующейся при деформации. Примесное торможение препятствует росту зерна при постдинамической рекристаллизации и способствует образованию более размерно однородной структуры на стадии частичной ДР. На стадии развитой ДР содержание примесей в меди не влияет на размер зерна.

Ключевые слова: деформация, медь, температурно-скомпенсированная скорость, динамическая рекристаллизаџия, постдинамическая рекристаллизация, структура.

\section{1. Введение}

В настоящее время большое внимание исследователей привлекают металлические материалы, в которых в результате воздействия большой пластической деформации, высокого давления и температуры получено структурно-фазовое состояние, обеспечивающее новые уникальные механические и физические свойства - прочностные, пластические, диффузионные, электрические и магнитные $[1,2]$. Одно из направлений получения уникального комплекса свойств - создание объемных субмикрокристаллических и наноструктурированных материалов. Несмотря на успехи, достигнутые в получении и исследовании таких материалов, многие вопросы изучены далеко не достаточно и остаются дискуссионными. Созданию таких материалов препятствуют наступление стадии насыщения накопления дефектов при фрагментации [3], ограничивающего измельчение структуры, и низкая термическая стабильность получаемых структур [4], приводящая к потере уникальных свойств. Поэтому изучение разных режимов и методов деформационно-термической обработки различных исходных материалов представляется весьма актуальным. Медь входит в состав новых композиционных материалов типа $\mathrm{Cu}-\mathrm{Nb}$, получаемые в результате сильной пластической деформации прокаткой, волочением или экструзией. Это новые перспективные высокопрочные материалы, сочетающие исключительно высокую механическую прочность с низким электросопротивлением [5]. Для дальнейшего прогресса в области создания и применения этих композитов в промышленных масштабах необходимо более глубокое понимание эволюции структуры как композитов в целом, так и их составляющих при термомеханической обработке, выявление закономерностей формирования текстуры, зеренной структуры и особенностей структуры границ раздела, которые определяют уникальные прочностные характеристики таких композитов. 
Большая пластическая деформация создает в материалах неравновесные структуры, приводя к изменению температуры, кинетики и полноты протекания структурных и фазовых превращений [6-10]. Так на $100-200{ }^{\circ} \mathrm{C}$ снижается температура рекристаллизации [11], что в ряде материалов при большой деформации может приводить к изменению структурообразующего процесса, например, деформационное упрочнение (наклеп) сменяется динамической рекристаллизацией (ДР). Такая смена струтурообразующих процессов является важным фактором, определяющим конечную структуру и свойства обрабатываемого материала, поэтому учитывается при моделировании деформационного поведения материала [12].

Ранее было установлено, что ДР оказывает влияние на эволюцию структуры меди высокой чистоты $(99,99$ мас. \%) в процессе деформации сдвигом под давлением при комнатной температуре [13]. Известно, что полнота протекания ДР определяется температурноскоростными условиями деформации $[14,15]$. Анализ температурно-скоростных условий деформации сдвигом под давлением позволил выявить стадийность структурообразования в условиях проявления ДР. Оказалось однако, что на формирование структуры высокочистой меди существенное влияние оказывает постдинамическая рекристаллизация (ПДР), приводящая к росту отдельных крупных зерен. Таким образом, структура меди высокой чистоты после окончания деформации продолжает непрерывно изменяться. Поэтому установить закономерности развития динамической рекристаллизации затруднительно. Известно, что легирование малым количеством примеси - эффективный способ торможения роста зерна [16]. Проведение деформации меди с разным содержанием примеси при температуре последующего вылеживания позволит выявить закономерности развития ДР и ПДР. Целью настоящей работы было исследование стадийности структурообразования в условиях проявления динамической рекристаллизации и роста зерна при ПДР в меди разной степени чистоты.

\section{2. Материал и методика}

Исследовали медь с разным содержанием примесей: особо чистую - 99,99 \%, бескислородную марки М0б (99,97 \%) и технической чистоты марки M1 (99,9 \%). Деформацию проводили методом сдвига под давлением 6 ГПа с углами поворота подвижной наковальни 15, 45, 60, 90 и 180 градусов и 1, 5 и 10 оборотов. Образцы для деформации вырезали в виде дисков диаметром 5 мм и толщиной 0,3 мм.

Для расчета истинной деформации использовали формулу [17]:

$$
e=e_{\text {сдвига }}+e_{\text {осадки }}=\ln \left(1+\left[\frac{\varphi^{*} r_{i}}{h_{i r}}\right]^{2}\right)^{0,5}+\ln \frac{h_{0}}{h_{i r}},
$$

где $\varphi$-угол поворота наковальни; $r_{i}$-расстояние от оси вращения; $h_{\boldsymbol{0}}$ и $h_{\boldsymbol{i}}$ - толщина образца до и после деформации на соответствующем $r_{i}$. Экспериментальная погрешность определения величины деформации составляла $\pm 0,2$ единицы истинной деформации. Максимальная деформация при сдвиге под давлением достигала $e=11$.

Формирование структуры при ДР идет в соответствии с температурой и скоростью деформации, совместное влияние которых учитывается параметром Зинера-Холломона (3$\mathrm{X).} \mathrm{На} \mathrm{практике} \mathrm{этот} \mathrm{параметр} \mathrm{представляют} \mathrm{в} \mathrm{виде}[15,18]$

$$
\ln \mathrm{Z}=\ln \dot{e}+\Delta H / R T,
$$

где $\dot{e}$ - истинная скорость деформации, $\mathrm{c}^{-1} ; \Delta H=107$ кДж/моль - энергия активации высокотемпературной деформации, для чистых металлов ее значение близко к энергии активации самодиффузии [19]; $R$ - газовая постоянная; $T$ - температура деформации, $300 \mathrm{~K}$.

Скорость деформации рассчитывали по формуле:

$$
\dot{e}=\Delta e / \Delta \tau,
$$

Orlova D. K. et al. / Effect of impurities on dynamic recrystallization in copper deformed in bridgman anvils. 
где $\Delta e$ - деформация, при которой произошло изменение структуры по сравнению с образцом, деформированным с меньшим углом поворота наковальни; $\Delta \tau$, с - время, за которое достигается соответствующее приращение степени деформации.

Структуру исследовали с помощью электронного микроскопа JEM 200CX на расстоянии 1,5 мм от центра образцов. Размер элементов структуры определяли по электронномикроскопическим светлопольным и темнопольным изображениям в рефлексе (111) $\gamma$ по результатам более 200 измерений с погрешностью менее 10 \% [20]. Строили гистограммы распределения элементов структуры по размерам, определяли максимальный $\left(d_{\max }\right)$ и средний $\left(d_{\mathrm{cp}}\right)$ размер элемента структуры, среднеквадратичное отклонение $(\sigma)$ и коэффициент вариации линейных размеров зерен $\left(K=\sigma / d_{\mathrm{cp}}\right)$. Время после деформации до исследования структуры не превышало двух суток.

\section{3. Результаты и их обсуждение}

Сопоставление результатов расчета температурно-скоростных условий деформации (рис. 1) и исследования структуры (рис. 2 и 3) показало, что при увеличении угла поворота наковальни независимо от содержания примесей последовательность процессов структурообразования одинаковая. Деформация с углом поворота наковальни $15^{\circ}$ проходит в условия $\ln Z>42$, электронно-микроскопическое исследование показало, что как в центре, так и на периферии образцов формируется однотипная структура горячего наклепа (рис. 2 a). Увеличение угла поворота наковальни до $45^{\circ}$ приводит к снижению $\ln Z<42$ и к началу ДР.
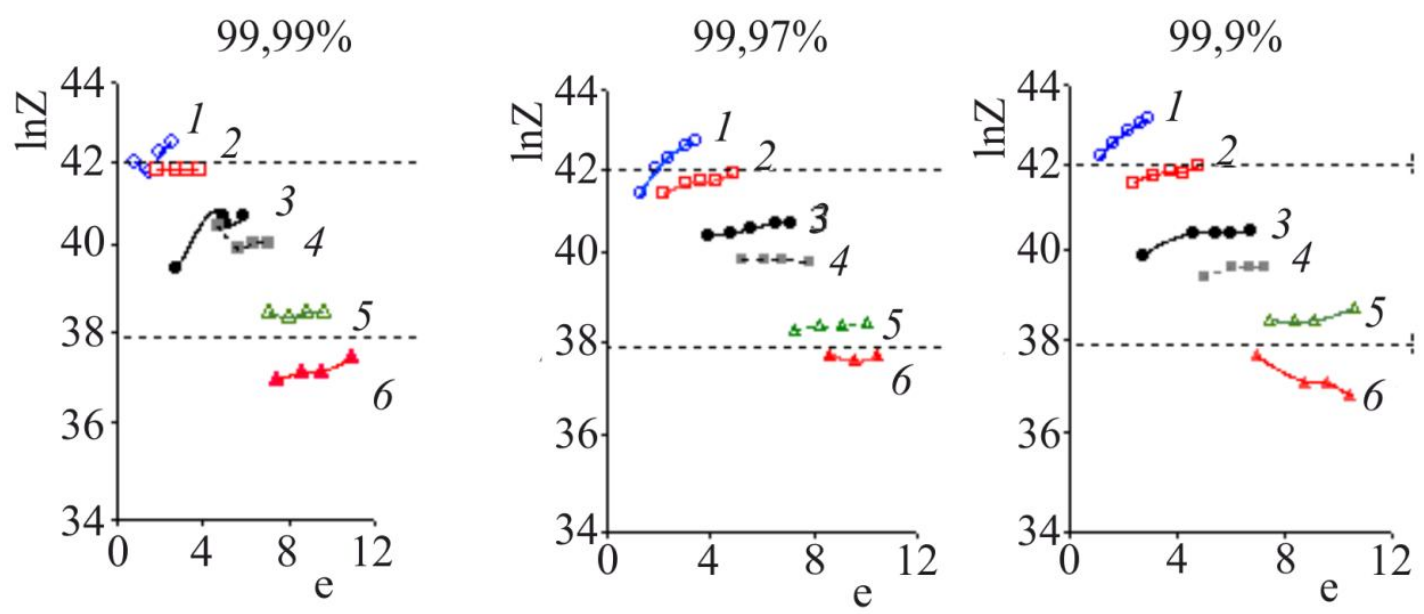

Рис.1 Температурно-скоростные условия деформации меди разной степени чистоты при деформации с различным углом поворота наковальни: $1-15^{\circ} ; 2-45^{\circ} ; 3-180^{\circ} ; 4-1$ оборот; 5 - 5 оборотов; $6-10$ оборотов

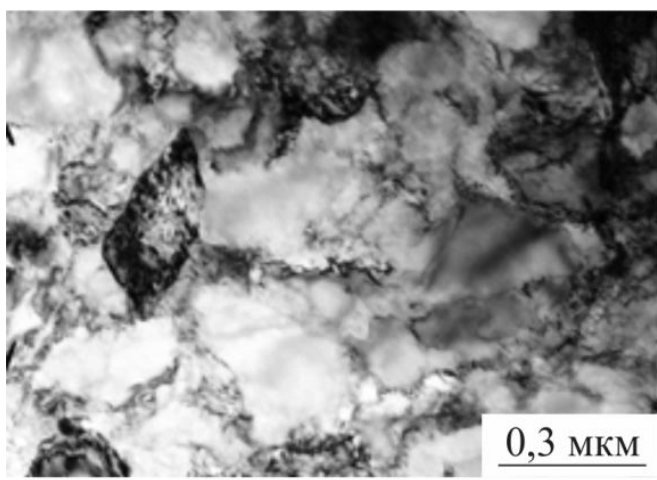

$a$

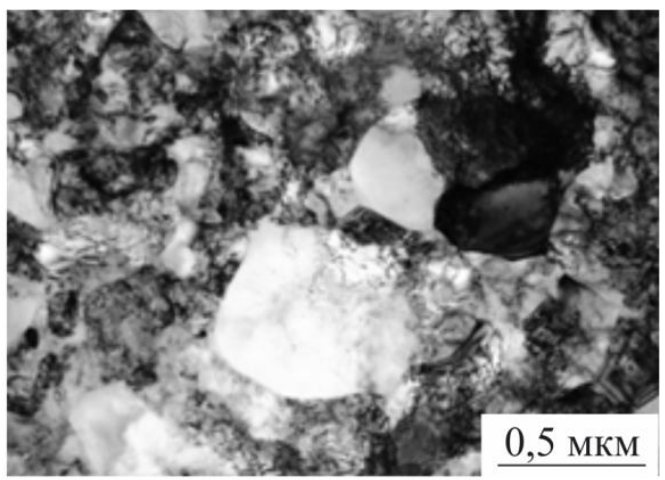

$\sigma$

Рис. 2. Микроструктура особо чистой меди:

$a$ - структура горячего наклепа $\ln Z>42 ; \sigma$ - первые ДР зерна $40<\ln Z<42$

Orlova D. K. et al. / Effect of impurities on dynamic recrystallization in copper deformed in bridgman anvils. 
Об этом можно судить по появлению первых динамически рекристаллизованных зерен (рис. 2 б). Такие зерна отделены от матрицы высокоугловой границей и в зависимости от времени возникновения либо не содержат дислокаций, либо в них разрешаются отдельные дислокации. Истинная деформация, необходимая для начала ДР, не зависимо от чистоты меди составляет $e \geq 2$ (рис. 1). При деформации с углом поворота $180^{\circ}(\ln Z=40)$ процессы деформационного упрочнения и динамической рекристаллизации развиваются в равной степени. Структура, сформированная в этих условиях, наиболее неустойчива, поскольку в ней наряду с динамически рекристаллизованными зернами, еще не претерпевшими наклеп и поэтому содержащими низкую плотность дислокаций, присутствует деформированная матрица с высокой плотностью дефектов, в которой ДР еще не произошла. Такого типа структура сохраняется при $\ln Z>38$. Стадия деформации в условиях $42>\ln Z>38$, таким образом, является стадией частичной ДР. В ходе деформации на 10 оборотов наковальни реализуется условие $\ln Z<38$, и ДР охватывает весь объем образца. В результате формируется субмикрозеренная структура, в которой зерна содержат разную плотность дислокаций в зависимости от времени их образования - это стадия развитой ДР.
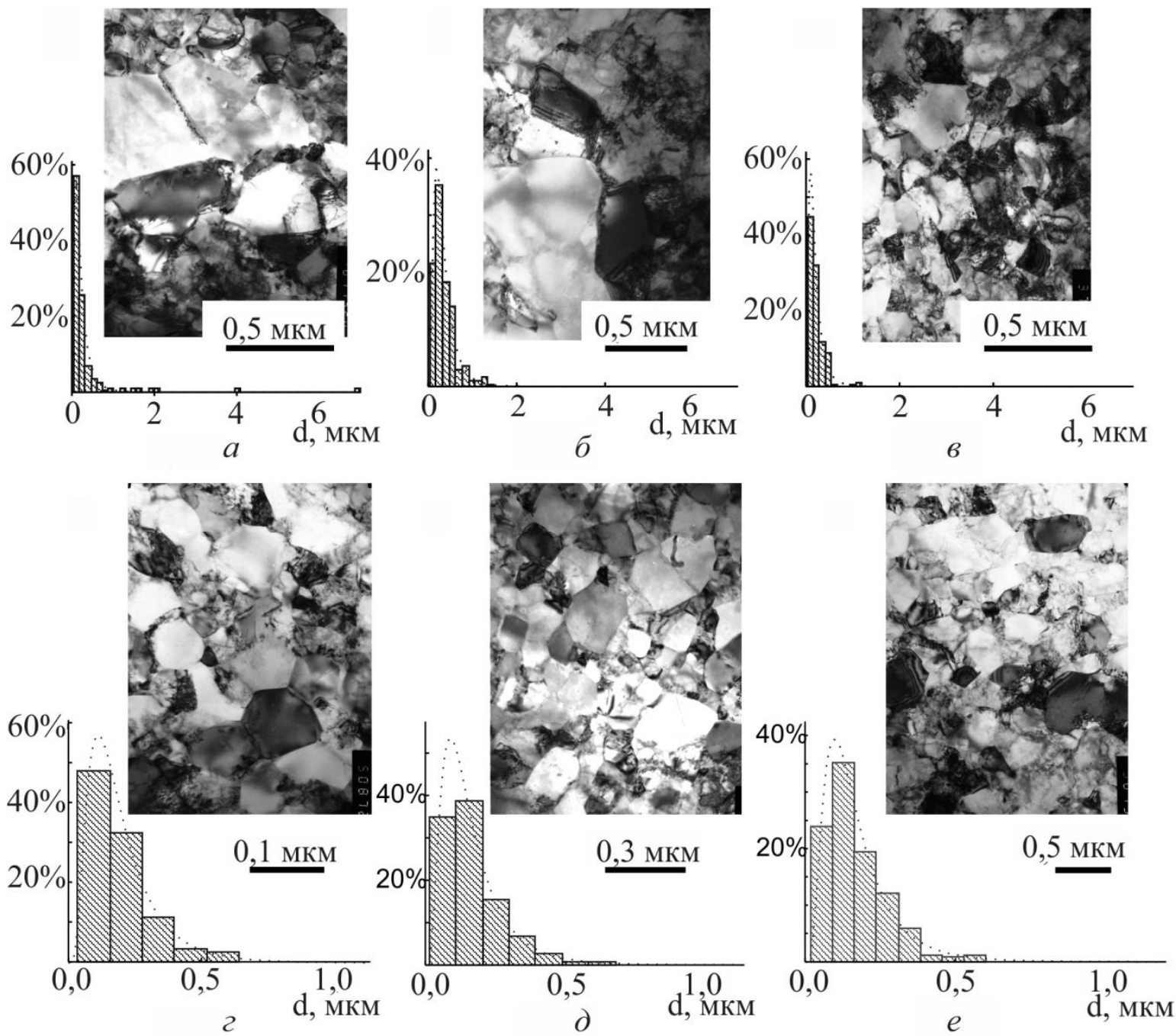

Рис.3. Гистограммы распределения элементов структуры по размерам и микроструктура меди, деформированной сдвигом под давлением на $180^{\circ}$, $\ln Z=40(a-8)$ и 10 оборотов; $\ln Z<38$ (2-e), чистотой: $a, z-99,99 \% ; \sigma, \partial-99,97 \% ; в, e-99,9 \%$ 
Более подробно исследовали влияние содержания примесей на структуру меди, полученную в условия $\ln Z=40$ и $\ln Z<38$. Независимо от содержания примесей и условий деформации в структуре наблюдаются как наклепанные зерна, так и зерна, свободные от дислокаций (рис. 3). Свободные от дислокаций зерна часто имеют правильную форму: тонкие прямые границы и уравновешенные тройные стыки. Такое совершенствование структуры могло произойти только по окончании деформации в ходе ПДР. После деформации в условиях $\ln Z=40$, зерен, способных к росту при ПДР, относительно немного, поэтому они имеют возможность, не сталкиваясь с другими зернами, расти до значительных размеров и не приобретают геометрически правильную форму (рис. $3 a-8$ ). В результате структура становится размерно неоднородной. Размеры ПДР зерен определяются содержанием примесей: для особо чистой меди $d_{\max }=7$ мкм, коэффициент вариации линейных размеров зерен составляет $K=2$, а для меди с большим содержанием примеси $d_{\max }=1,0-1,5$ мкм и $K=1$. Полученные значения коэффициента вариации свидетельствует о сильной размерной неоднородности структуры [21], уменьшающейся с увеличением содержания примесей. На стадии развитой ДР (рис. 3 2-e) плотность способных к росту зерен велика, и их взаимное столкновение обусловливает геометрически правильную форму и заметно меньший размер наиболее крупных зерен. На стадии развитой ДР максимальный размер зерна не превышает 0,6-0,7 мкм и, как оказалось, не зависит от содержания примесей в меди. На этой стадии формируется размерно-однородная структура $(K=0,5-0,6[21])$.

\section{4. Заключение}

В меди, деформированной сдвигом под давлением при комнатной температуре, независимо от содержания примесей от 0,01 до $0,1 \%$ по достижении истинной деформации е $=2$ начинается динамическая рекристаллизация. Полнота протекания динамической рекристаллизации и, соответственно, тип формирующейся структуры определяются температурноскоростными условиями деформации $(\ln Z)$ независимо от содержания примесей.

Выделены интервалы параметра Зинера-Холломона $\ln Z$, соответствующие структурам разного типа. В интервале $35<\ln Z<38$ динамическая рекристаллизация проходит во всем объеме материала (стадия развитой динамической рекристаллизации), при $38<\ln Z<42$ объемная доля структуры, претерпевшей динамическую рекристаллизацию, уменьшается с увеличением $\ln Z$ (стадия частичной динамической рекристаллизации). При $\ln Z>42$ признаков динамической рекристаллизации не обнаружено (стадия наклепа).

Влияние содержания примесей в меди на развитие постдинамической рекристаллизации различно на разных стадиях структурных состояний. На стадии частичной динамической рекристаллизации примесное торможение препятствует росту зерна при постдинамической рекристаллизации и способствует образованию более размерно однородной структуры. На стадии развитой динамической рекристаллизации содержание примесей не влияет на размер зерна и размерную однородность структуры.

\section{Благодарность}

Работа выполнена в рамках государственного задания ФАНО России (тема «Кристалл» №01201463333) при частичной поддержке проекта УрО РАН №15-17-2-11. Электронно-микроскопическое исследование выполнено в ЦКП «Испытательный центр нанотехнологий и перспективных материалов» ИФМ УрО РАН.

\section{Литература:}

1. Валиев Р. 3., Александров И. В. Наноструктурные материалы, полученные интенсивной пластической деформацией. - М. : Логос, 2000. - 272 с.

2. Andrievsky R. A., Glezer A. M. Size effects in nanocrystalline materials: II. Mechanical and physical properties // Physics of Metals and Metallography. - 2000. - Vol. 89, iss. 1. - P. 83-102.

Orlova D. K. et al. / Effect of impurities on dynamic recrystallization in copper deformed in bridgman anvils. 
3. Теплов В. А., Пилюгин В. П., Талуц Г. Г. Образование диссипативной структуры и фазовые переходы в сплавах железа при сдвиге под давлением // Известия РАН. Металлы. 1992. - № 2. - C. 109-154.

4. Носкова Н. И., Мулюков Р. Р. Субмикрокристаллические и нанокристаллические металлы и сплавы. - Екатеринбург: УрО РАН, 2003. - 279 с.

5. Specific Features of Interfaces in $\mathrm{Cu}-\mathrm{Nb}$ Nanocomposites / E. N. Popova, I. L. Deryagina, E. G. Valova-Zaharevskaya, A. V. Stolbovsky, N. E. Khlebova, V. I. Pantsyrny // Defect and Diffusion Forum. - 2014. - Vol. 354. - P. 183-188. - DOI: 10.4028/www.scientific.net/DDF.354.183

6. Influence of the relaxation processes on the structure formation in pure metals and alloys under high-pressure deformation / M. V. Degtyarev, T. I. Chashchukhina, L. M. Voronova, A. M. Patselov, V. P. Pilyugin // Acta Materialia. - 2007. - Vol. 55. - P. 6039-6050. DOI: 10.1016/j.actamat.2007.04.017.

7. Structure and mechanical properties of aging Al-Li-Cu-Zr-Sc-Ag alloy after severe plastic deformation by high-pressure torsion / L. I. Kaigorodova, D. Yu. Rasposienko, V. G. Pushin, V. P. Pilyugin, S. V. Smirnov // Physics of Metals and Metallography. - 2015. - Vol. 116, iss. 4. P. 346-355. - DOI: 10.1134/S0031918X15040080.

8. Mossbauer analysis of deformation dissolution of the products of cellular decomposition in high-nitrogen chromium manganese austenite steel / V. A. Shabashov, L. G. Korshunov., V. V. Sagaradze, N. V. Kataeva, A. E. Zamatovsky, A. V. Litvinov, K. A. Lyashkov // Philosophical Magazine. - 2014. - Vol. 94. - P. 668-682. - DOI: 10.1080/14786435.2013.859758.

9. Structure and microhardness of chromium-zirconium bronze subjected to severe plastic deformation by dynamic channel-angular pressing and rolling / V. I. Zel'dovich, N. Yu. Frolova, I. V. Khomskaya, A. E. Kheifets, E. V. Shorokhov, P. A. Nasonov // Physics of Metals and Metallography. - 2014. - Vol. 115, iss. 5. - P. 465-470. - DOI: 10.1134/S0031918X14050159.

10. Structure of an AMts aluminum alloy after high-pressure torsion in liquid nitrogen / I. G. Brodova, I. G. Shirinkina, A. N. Petrova, V. P. Pilyugin, T. P. Tolmachev // Physics of Metals and Metallography. - 2013. - Vol. 114, iss. 8. - P. 667-671. - DOI: 10.1134/S0031918X13080024.

11. Features of low-temperature recrystallization of nickel and copper / N. A. Smirnova, V. I. Levit, V. P. Pilyugin, R. I. Kuznetsov, M. V. Degtyarev // Physics of Metals and Metallography. 1986. - Vol. 62, iss. 3. - P. 140-144.

12. Smirnov A. S., Konovalov A. V., Muizemnek O. Yu. Modelling and Simulation of Strain Resistance of Alloys Taking into Account Barrier Effects // Diagnostics, Resource and Mechanics of materials and structures. - 2015. - Iss. 1. - P. 61-72. URL: $\quad$ http://dream-journal.org/issues/2015-1/2015-1_18.html (accessed: 10.09.2015). DOI: $10.17804 / 2410-9908.2015 .1 .061-072$.

13. Dynamic recrystallization in copper deformed by shear under pressure / T. I. Chashchukhina, M. V. Degtyarev, M. Yu. Romanova, L. M. Voronova // Physics of Metals and Metallography. -2004. - Vol. 98. - P. 639-647.

14. Диаграммы горячей деформации, структура и свойства сталей / М. Л. Бернштейн, С. В. Добаткин, Л. М. Капуткина, С. Д. Прокошкин. - М. : Металлургия, 1989. - 544 с.

15. Левит В. И., Смирнов М. А. Высокотемпературная термомеханическая обработка аустенитных сталей и сплавов. - Челябинск : ЧГТУ, 1995. - 276с.

16. Горелик С. С. Рекристаллизация металлов и сплавов. - М. : Металлургия, 1978. - 568 с.

17. Установление соответствия между степенью деформации, твердостью и размерами элементов структуры железа и конструкционных сталей при большой пластической деформации различными способами / М. В. Дегтярев, Т. И. Чащухина, Л. М. Воронова, В. И. Копылов // Физическая мезомеханика. - 2013. - Т.16, № 6. - С. 71-80.

18. McQeen H. J. The production and utility of recovered dislocation substructures / Metallurgical Transactions A. - 1977. - Vol. 8, iss. 6. - P. 807-824. - DOI: 10.1007/BF02661562. 
open-access journal

19. Amirkhanov N. M., Islamgaliev R. K., Valiev R. Z. / Thermal relaxation and grain growth upon isothermal annealing of ultrafine grained copper produced by severe plastic deformation // Physics of Metals and Metallography. - 1998. - Vol. 86, iss. 3. - P. 296-301.

20. Салтыков С. А. Стереометрическая металлография. - М. : Металлургия, 1970. - 375 с.

21. Новиков В. Ю. Вторичная рекристаллизация. - М. : Металлургия, 1990. - 128c. 